\title{
Quasi-long-range order in the random anisotropy Heisenberg model: functional renormalization group in $4-\epsilon$ dimensions
}

\author{
D.E. Feldman \\ Department of Condensed Matter Physics, Weizmann Institute of Science, 76100 Rehovot, Israel \\ Landau Institute for Theoretical Physics, 142432 Chernogolovka, Moscow region, Russia
}

\begin{abstract}
The large-distance behaviors of the random field and random anisotropy $O(N)$ models are studied with the functional renormalization group in $4-\epsilon$ dimensions. The random anisotropy Heisenberg $(N=3)$ model is found to have a phase with an infinite correlation length at low temperatures and weak disorder. The correlation function of the magnetization obeys a power $\operatorname{law}\left\langle\mathbf{m}\left(\mathbf{r}_{1}\right) \mathbf{m}\left(\mathbf{r}_{2}\right)\right\rangle \sim$ $\left|\mathbf{r}_{1}-\mathbf{r}_{2}\right|^{-0.62 \epsilon}$. The magnetic susceptibility diverges at low fields as $\chi \sim H^{-1+0.15 \epsilon}$. In the random field $O(N)$ model the correlation length is found to be finite at the arbitrarily weak disorder for any $N>3$. The random field case is studied with a new simple method, based on a rigorous inequality. This approach allows one to avoid the integration of the functional renormalization group equations.
\end{abstract}

75.10 Nr, 75.50 Kj, 64.60 Cn

\section{INTRODUCTION}

The effect of impurities on the order in condensed matter is interesting, since the disorder is almost inevitably present in any system. If the disorder is weak the short-range order is the same as in the pure system. However, the large-distance behavior can be strongly modified by the arbitrarily weak disorder. This happens in the systems of continuous symmetry in the presence of the random symmetry breaking field [1]. The first experimental example of this kind is the amorphous magnet [2,3]. During the last decade a lot of other related objects were found. These are liquid crystals in the porous media [4], nematic elastomers [5], He-3 in aerogel [6] and vortex phases of impure superconductors [7]. The nature of the low-temperature phases of these systems is still unclear. The only reliable statement is that a long-range order is absent [1, 10]. However, other details of the large-distance behavior are poorly understood.

The neutron scattering [11] reveals sharp Bragg peaks in impure superconductors at low temperatures and weak external magnetic fields. Since the vortices can not form a regular lattice [8] it is tempting to assume that there is a quasi-long-range order (QLRO), that is the correlation length is infinite and correlation functions depend on the distance slow. Recent theoretical [12,13] and numerical [14] studies of the random field XY model, which is the simplest model of the vortex system in the impure superconductor [7], support this picture. The theoretical advances 12.13] are afforded by two new technical approaches: the functional renormalization group [15] and the replica variational method [16]. These methods are free from drawbacks of the standard renormalization group and give reasonable results. The variational method regards the possibility of spontaneous replica symmetry breaking and treats the fluctuations approximately. On the other hand the functional renormalization group provides a subtle analysis of the fluctuations about the replica symmetrical ground state. Surprisingly, the methods give close and sometimes even the same results.

Both techniques were originally suggested for the random manifolds 115,16 and then allowed to obtain information about some other disordered systems with the Abelian symmetry [12,13, 17 19]. Less is known about the non-Abelian systems. The simplest of them are the random field (RF) [1] and random anisotropy (RA) [2] Heisenberg models. The latter was introduced as a model of the amorphous magnet [2, 3]. In spite of a long discussion, initiated by Ref. 20], the question of QLRO in these models is still open. There is an experimental evidence in favor of no QLRO [21]. On the other hand recent numerical simulations [22] support the possibility of QLRO in these systems. The only theoretical approach, developed up to now, is based on the spherical approximation 9, 23,24, and predicts the absence of QLRO at $N \gg 1$ magnetization components. However, there is no reason for this approximation to be valid at $N \sim 1$.

In this paper we study the RF and RA $O(N)$ models in $4-\epsilon$ dimensions with the functional renormalization group. The large-distance behaviors of the systems are found to be quite different. Whereas in the RF $O(N)$ model with $N>3$ the correlation length is always finite, the RA Heisenberg $(N=3)$ model has a phase with QLRO. In that phase the correlation function of the magnetization obeys a power law and the magnetic susceptibility diverges at low fields.

The paper has the following structure. In the second section the models are formulated and a qualitative discussion is given. The third section contains a derivation of the one-loop renormalization group (RG) equations. The 4th 
section is devoted to the RF model. The absence of QLRO in that model at $N>3$ is shown with a new method, based on a rigorous inequality. This approach simplifies tedious RG calculations and can be useful in other problems. The RA case is considered in the 5th section. The stable RG fixed point corresponds to QLRO in $4-\epsilon$ dimensions at $N \sim 1$. In particular, at the weak disorder the correlation length is infinite in the low temperature phases of the RA XY $(N=2)$ and Heisenberg $(N=3)$ models. However, QLRO is absent at $N \geq 10$. In 4 dimensions the correlation functions of the RA Heisenberg model depend on the distance logarithmically. The exact result for the two-spin correlator is given by the $\ln ^{-0.62} R$ law. The Conclusion contains a discussion of the results. Appendix A is devoted to a generalization of the Schwartz-Soffer inequality [25]. The generalized inequality is applied to the stability analyses of the RG fixed points. Appendix B describes a simple Migdal-Kadanoff renormalization group approach that reproduces qualitatively the results of the rigorous method. This approximation provides good estimations of the critical exponents of the RA XY and Heisenberg models. Appendix C includes some technical details of the functional RG in the spherical model.

\section{MODEL}

To describe the large-distance behavior at low temperatures we use the classical nonlinear $\sigma$-model with the Hamiltonian

$$
H=\int d^{D} x\left[J \sum_{\mu} \partial_{\mu} \mathbf{n}(\mathbf{x}) \partial_{\mu} \mathbf{n}(\mathbf{x})+V_{\mathrm{imp}}(\mathbf{x})\right],
$$

where $\mathbf{n}(\mathbf{x})$ is the unit vector of the magnetization, $V_{\mathrm{imp}}(\mathbf{x})$ the random potential. In the RF case it has the form

$$
V_{\mathrm{imp}}=-\sum_{\alpha} h_{\alpha}(\mathbf{x}) n_{\alpha}(\mathbf{x}) ; \alpha=1, \ldots, N,
$$

where the random field $\mathbf{h}(\mathbf{x})$ has a Gaussian distribution and $\left\langle h_{\alpha}(\mathbf{x}) h_{\beta}\left(\mathbf{x}^{\prime}\right)\right\rangle=A^{2} \delta\left(\mathbf{x}-\mathbf{x}^{\prime}\right) \delta_{\alpha \beta}$. In the RA case the random potential is given by the equation

$$
V_{\mathrm{imp}}=-\sum_{\alpha, \beta} \tau_{\alpha \beta}(\mathbf{x}) n_{\alpha}(\mathbf{x}) n_{\beta}(\mathbf{x}) ; \alpha, \beta=1, \ldots, N,
$$

where $\tau_{\alpha \beta}(\mathbf{x})$ is a Gaussian random variable, $\left\langle\tau_{\alpha \beta}(\mathbf{x}) \tau_{\gamma \delta}\left(\mathbf{x}^{\prime}\right)\right\rangle=A^{2} \delta_{\alpha \gamma} \delta_{\beta \delta} \delta\left(\mathbf{x}-\mathbf{x}^{\prime}\right)$. The random potential (2.3) corresponds to the same symmetry as the more conventional choice $V_{\mathrm{imp}}=-(\mathbf{h n})^{2}$ but is more convenient for the further discussion.

We assume that the temperature is low and the thermal fluctuations are negligible. The Imry-Ma argument [1]:9] suggests that in our problem the long-range order is absent at any dimension $D<4$. One can estimate the Larkin length, up to which there are strong ferromagnetic correlations, with the following qualitative RG approach. Let one remove the fast modes and rewrite the Hamiltonian in terms of the block spins, corresponding to the scale $L=b a$, where $a$ is the ultraviolet cut-off, $b>1$. Then let one make rescaling such that the Hamiltonian would restore its initial form with new constants $A(L), J(L)$. Dimensional analysis provides estimations

$$
J(L) \sim b^{D-2} J(a) ; A(L) \sim b^{D / 2} A(a)
$$

To estimate the typical angle $\phi$ between neighbor block spins, one notes that the effective field, acting on each spin, has two contributions: the exchange contribution and the random one. The exchange contribution of order $J(L)$ is oriented along the local average direction of the magnetization. The random contribution of order $A(L)$ may have any direction. This allows one to write at low temperatures that $\phi(L) \sim A(L) / J(L)$. The Larkin length corresponds to the condition $\phi(L) \sim 1$ and equals $L \sim(J / A)^{2 /(4-D)}$ in agreement with the Imry-Ma argument [1]. If Eq. (2.4) were exact the Larkin length could be interpreted as the correlation length. However, there are two sources of corrections to Eq. (2.4). Both of them are relevant already at the derivation of the RG equation for the pure system in $2+\epsilon$ dimensions [26]. The first source is the renormalization due to the interaction and the second one results from the magnetization rescaling which is necessary to ensure the fixed length condition $\mathbf{n}^{2}=1$. The leading corrections to Eq. (2.4) are proportional to $\phi^{2} J, \phi^{2} A$. Thus, the RG equation for the combination $(A(L) / J(L))^{2}$ reads

$$
\frac{d}{d \ln L}\left(\frac{A(L)}{J(L)}\right)^{2}=\epsilon\left(\frac{A(L)}{J(L)}\right)^{2}+c\left(\frac{A(L)}{J(L)}\right)^{4}, \epsilon=4-D
$$


If the constant $c$ in Eq. (2.5) is positive the Larkin length is the correlation length indeed. But if $c<0$ the RG equation has a fixed point, corresponding to the phase with an infinite correlation length. As seen below, both situations are possible, depending on the system.

The large-distance behaviors of the RF and RA $O(N)$ models are known in two limit cases: $N=2$ and $N=\infty$. In the spherical limit $(N=\infty)$ QLRO is absent (Appendix C, 23]) while the XY model possesses QLRO [12,13, 27]. Hence, the ordering disappears at some critical number $N_{c}$ of the magnetization components. This critical number is larger in the RA model, since the fluctuations of the magnetization are stronger in the RF case. Indeed, in the RF model the magnetization tends to be oriented along the random field, whereas in the RA case there are two preferable local magnetization directions so that the spins tend to lie in the same semispace.

\section{RG EQUATIONS}

In the previous section the RG equations are discussed from the qualitative point of view. Eq. (2.5) corresponds to the Migdal-Kadanoff approach of Appendix B. In the present section we derive the RG equations in a systematic way.

The one-loop RG equations for the $N$-component RF and RA models in $4+\epsilon$ dimensions were already derived in Ref. [28]. We can directly use that result, since the RG equations in dimensions $D<4$ can be obtained by just changing the sign of $\epsilon$. However, the approach 28] is cumbersome and we provide below a simpler derivation.

We use the method, suggested by Polyakov 26] for the pure system in $2+\epsilon$ dimensions. This method is technically simpler and closer to the intuition than the other approaches. A disadvantage of the method is the difficulty of the generalization for the higher orders in $\epsilon$. This generalization requires the field-theoretical approach [29].

The same consideration as in the XY [13] and random manifold [15] models suggests that near a zero-temperature fixed point in $4-\epsilon$ dimensions there is an infinite set of relevant operators. Let us show that after the replica averaging the relevant part of the effective replica Hamiltonian can be represented in the form

$$
H_{R}=\int d^{D} x\left[\sum_{a} \frac{1}{2 T} \sum_{\mu} \partial_{\mu} \mathbf{n}_{a} \partial_{\mu} \mathbf{n}_{a}-\sum_{a b} \frac{R\left(\mathbf{n}_{a} \mathbf{n}_{b}\right)}{T^{2}}\right]
$$

where $a, b$ are replica indices, $R(z)$ is some function, $T$ the temperature. We ascribe to the field $\mathbf{n}$ the scaling dimension 0 . We also assume that the coefficient before the gradient term in (3.1) is $1 /(2 T)$ at any scale. Then in the $(4-\epsilon)$-dimensional space the scaling dimension of the temperature $\Delta_{T}=-2+O(\epsilon)$. Any operator $A$ containing $m$ different replica indices is proportional [15] to $1 / T^{m}$. Hence, the scaling dimension $\Delta_{A}$ of the operator $A$ satisfies the relation $\Delta_{A}=4-n+m \Delta_{T}+O(\epsilon)$, where $n$ is the number of the spatial derivatives in the operator. The relevant operators have $\Delta_{A} \geq 0$. Hence, the relevant operators can not contain more than two different replica indices. A symmetry consideration shows that all the possible relevant operators are included into Eq. (3.1). The function $R(z)$ is arbitrary in the RF case. In the RA case $R(z)$ is even due to the symmetry with respect to changing the sign of the magnetization.

The one-loop RG equations for the $N$-component model in $4-\epsilon$ dimensions are obtained by a straightforward combination of the methods of Refs. [15] and [26]. We express each replica $\mathbf{n}^{a}(\mathbf{x})$ of the magnetization as a combination of fast fields $\phi_{i}^{a}(\mathbf{x}), i=1, \ldots, N-1$ and a slow field $\mathbf{n}^{\prime a}(\mathbf{x})$ of the unit length. We use the representation

$$
\mathbf{n}^{a}(\mathbf{x})=\mathbf{n}^{\prime a}(\mathbf{x}) \sqrt{1-\sum_{i}\left(\phi_{i}^{a}(\mathbf{x})\right)^{2}}+\sum_{i} \phi_{i}^{a}(\mathbf{x}) \mathbf{e}_{i}^{a}(\mathbf{x}),
$$

where the unit vectors $\mathbf{e}_{i}^{a}(\mathbf{x})$ are perpendicular to each other and the vector $\mathbf{n}^{\prime a}(\mathbf{x})$. The slow field $\mathbf{n}^{\prime a}$ can be chosen in different ways. For example, one can cut the system into blocks of size $L \gg a$, where $a$ is the ultra-violet cut-off. In the center $\mathbf{x}$ of a block the vector $\mathbf{n}^{\prime a}(\mathbf{x})$ should be parallel to the total magnetization of the block. In the other points the field $\mathbf{n}^{\prime a}$ should be interpolated. We assume that the fluctuations of the magnetization are weak, that is $\left\langle\phi_{i}^{2}\right\rangle \ll 1$. Then the fluctuations of the field $\mathbf{n}^{a}$ are orthogonal to the vector $\mathbf{n}^{\prime a}$ because of the fixed length constraint $\left(\mathbf{n}^{a}\right)^{2}=1$.

To substitute the expression (3.2) into the Hamiltonian we have to differentiate the vectors $\mathbf{e}_{i}^{a}$. We use the following definition

$$
\frac{\partial \mathbf{e}_{i}^{a}}{\partial x_{\mu}}=-c_{\mu i}^{a} \mathbf{n}^{\prime a}+\sum_{k} f_{\mu, i k}^{a} \mathbf{e}_{k}^{a}
$$


It is easy to show [26] that $\sum_{\mu i}\left(c_{\mu i}^{a}\right)^{2}=\sum_{\mu}\left(\partial_{\mu} \mathbf{n}^{\prime a}\right)^{2}$. With the accuracy up to the second order in $\phi$ the replica Hamiltonian (3.1) can be represented as follows

$$
\begin{array}{r}
H_{R}=\int d^{D} x\left[\frac{1}{2 T} \sum_{a}\left\{\left(\partial_{\mu} \mathbf{n}^{a}\right)^{2}\left(1-\left(\phi_{i}^{a}\right)^{2}\right)+c_{\mu i}^{a} c_{\mu k}^{a} \phi_{i}^{a} \phi_{k}^{a}+\left(\partial_{\mu} \phi_{i}^{a}-f_{\mu, i k}^{a} \phi_{k}^{a}\right)^{2}\right\}\right. \\
\left.-\frac{1}{T^{2}} \sum_{a b}\left\{R\left(\mathbf{n}^{\prime a} \mathbf{n}^{\prime b}\right)+A^{a b}\left(\phi_{i}^{a}\right)^{2}+B_{i j}^{a b} \phi_{i}^{a} \phi_{j}^{a}+C_{i j}^{a b} \phi_{i}^{a} \phi_{j}^{b}\right\}\right],
\end{array}
$$

where the summation over the repeated indices $i, j, k, \mu$ is assumed and

$$
\begin{array}{r}
A^{a b}=-\left(\mathbf{n}^{\prime a} \mathbf{n}^{\prime b}\right) R^{\prime}\left(\mathbf{n}^{\prime a} \mathbf{n}^{\prime b}\right) ; B_{i j}^{a b}=\left(\mathbf{n}^{\prime b} \mathbf{e}_{i}^{a}\right)\left(\mathbf{n}^{\prime b} \mathbf{e}_{j}^{a}\right) R^{\prime \prime}\left(\mathbf{n}^{\prime a} \mathbf{n}^{\prime b}\right) ; \\
C_{i j}^{a b}=\left(\mathbf{e}_{i}^{a} \mathbf{e}_{j}^{b}\right) R^{\prime}\left(\mathbf{n}^{\prime a} \mathbf{n}^{\prime b}\right)+\left(\mathbf{n}^{\prime a} \mathbf{e}_{j}^{b}\right)\left(\mathbf{n}^{\prime b} \mathbf{e}_{i}^{a}\right) R^{\prime \prime}\left(\mathbf{n}^{\prime a} \mathbf{n}^{\prime b}\right) .
\end{array}
$$

In the last formula $R^{\prime}$ and $R^{\prime \prime}$ denote the first and second derivatives of the function $R(z)$. We have omitted the terms of the first order in $\phi$ in Eq. (3.4). These terms are proportional to the products of the fast field $\phi$ and some slow fields. Hence, they are non-zero only in narrow shells of the momentum space. One can show that their contributions to the $\mathrm{RG}$ equations are negligible.

To obtain the RG equations we have to integrate out the fast variables $\phi_{i}^{a}$. Near a zero-temperature fixed point the Jacobian of the transformation $\mathbf{n} \rightarrow\left(\mathbf{n}^{\prime}, \phi_{i}\right)$ can be ignored, since the Jacobian does not depend on the temperature. The integration measure is determined from the condition that the field $\mathbf{n}^{\prime a}$ is a slow part of the magnetization. This condition imposes restrictions on the fields $\phi$. The expression (3.4) depends on the choice of the vectors $\mathbf{e}_{i}^{a}$ (3.2). However, after integrating out the fields $\phi$ the Hamiltonian can depend only on the slow part $\mathbf{n}^{\prime a}$ of the magnetization. One can make the calculations simpler, considering special realizations of the field $\mathbf{n}^{\prime a}$. To find the renormalization of the disorder-induced term $R(z)$ (3.1) we can assume that the field $\mathbf{n}^{\prime a}$ does not depend on the coordinates. The renormalization of the gradient energy can be determined, assuming that the vectors $\mathbf{n}^{\prime a}(\mathbf{x})$ depend on one spatial coordinate only and lie in the same plane. In both cases the vectors $\mathbf{e}_{i}^{a}$ can be chosen such that in Eq. (3.3) $f_{\mu, i k}^{a}=0$. At such a choice the integration measure can be omitted and the fields $\phi_{i}^{a}$ can be considered as weakly interacting fields with the wave vectors from the interval $1 / a>q>1 / L$.

To derive the one-loop RG equations we express the free energy via the Hamiltonian (3.4). Then we expand the exponent in the partition function up to the second order in $\left(H_{R}-\int d^{D} x \sum_{\mu i}\left(\partial_{\mu} \phi_{i}\right)^{2} /(2 T)\right)$ and integrate over $\phi_{i}^{a}$. Finally, we make a rescaling. The vectors $\mathbf{e}_{i}^{a}$ can be excluded from the final expressions with the relation $\sum_{i}\left(\mathbf{a e}_{i}^{a}\right)\left(\mathbf{b e}_{i}^{a}\right)=(\mathbf{a b})-\left(\mathbf{a n}^{\prime a}\right)\left(\mathbf{b n}^{\prime a}\right)$. In a zero-temperature fixed point the RG equations are

$$
\begin{gathered}
\frac{d \ln T}{d \ln L}=-(D-2)+2(N-2) R^{\prime}(1)+O\left(R^{2}, T\right) \\
0=\frac{d R(z)}{d \ln L}=\epsilon R(z)+4(N-2) R(z) R^{\prime}(1)-2(N-1) z R^{\prime}(1) R^{\prime}(z)+2\left(1-z^{2}\right) R^{\prime}(1) R^{\prime \prime}(z) \\
+\left(R^{\prime}(z)\right)^{2}\left(N-2+z^{2}\right)-2 R^{\prime}(z) R^{\prime \prime}(z) z\left(1-z^{2}\right)+\left(R^{\prime \prime}(z)\right)^{2}\left(1-z^{2}\right)^{2},
\end{gathered}
$$

where the factor $1 /\left(8 \pi^{2}\right)$ is absorbed into $R(z)$ to simplify notations. The RG equations become simpler after the substitution of the argument of the function $R(z): z=\cos \phi$. In terms of this new variable one has to find even periodic solutions $R(\phi)$. The period is $2 \pi$ in the RF case and $\pi$ in the RA case due to the additional symmetry of the RA model. The one-loop equations get the form

$$
\begin{gathered}
\frac{d \ln T}{d \ln L}=-(D-2)-2(N-2) R^{\prime \prime}(0)+O\left(R^{2}, T\right) ; \\
0=\frac{d R(\phi)}{d \ln L}=\epsilon R(\phi)+\left(R^{\prime \prime}(\phi)\right)^{2}-2 R^{\prime \prime}(\phi) R^{\prime \prime}(0)- \\
(N-2)\left[4 R(\phi) R^{\prime \prime}(0)+2 \operatorname{ctg} \phi R^{\prime}(\phi) R^{\prime \prime}(0)-\left(\frac{R^{\prime}(\phi)}{\sin \phi}\right)^{2}\right]+O\left(R^{3}, T\right)
\end{gathered}
$$

Eq. (3.8) provides the following result for the scaling dimension $\Delta_{T}$ of the temperature

$$
\Delta_{T}=-2+\epsilon-2(N-2) R^{\prime \prime}(0) .
$$


The two-spin correlation function is given in the one-loop order [26] by the expression

$$
\left\langle\mathbf{n}^{a}(\mathbf{x}) \mathbf{n}^{a}\left(\mathbf{x}^{\prime}\right)\right\rangle=\left\langle\mathbf{n}^{\prime a}(\mathbf{x}) \mathbf{n}^{\prime a}\left(\mathbf{x}^{\prime}\right)\right\rangle\left(1-\left\langle\sum_{i}\left(\phi_{i}^{a}\right)^{2}\right\rangle\right) .
$$

Hence, in the fixed point $\left\langle\mathbf{n}(\mathbf{x}) \mathbf{n}\left(\mathbf{x}^{\prime}\right)\right\rangle \sim\left|\mathbf{x}-\mathbf{x}^{\prime}\right|^{-\eta}$, where

$$
\eta=-2(N-1) R^{\prime \prime}(\phi=0)
$$

Let us find the magnetic susceptibility in the weak uniform external field $H$. We add to the Hamiltonian (3.1) the term $-\sum_{a} \int d^{D} x H n_{z}^{a} / T$ (the field is directed along the z-axis). The renormalization of the field $H$ is determined by the renormalization of the temperature $(3.8)$ and the field $\mathbf{n}$. In the zero-loop order the renormalized magnetic field $h(L)$ depends on the scale as $h(L)=H \times(L / a)^{2}$. Hence, the correlation length $R_{c} \sim H^{-1 / 2}$. The magnetization, averaged over a block of size $R_{c}$, is oriented along the field. The absolute value of this average magnetization is proportional to $R_{c}^{-\eta / 2}$. This allows us to calculate the critical exponent $\gamma$ of the magnetic susceptibility $\chi(H) \sim H^{-\gamma}$ in a zero-temperature fixed point:

$$
\gamma=1+(N-1) R^{\prime \prime}(\phi=0) / 2 .
$$

In Ref. [28] Eqs. (3.6, 3.7) were derived with a different method. In that paper the critical behavior in $4+\epsilon$ dimensions was studied by considering analytical fixed point solutions $R(z)$. In the Heisenberg model, analytical solutions are absent and they are unphysical for $N \neq 3$ [28]. In $4-\epsilon$ dimensions appropriate analytical solutions are absent for any $N$. To demonstrate this let us differentiate Eq. (3.7) over $z$ at $z=1$. For any analytical $R(z)$ we obtain the following flow equation

$$
\frac{d R^{\prime}(z=1)}{d \ln L}=\epsilon R^{\prime}(z=1)+2(N-2)\left(R^{\prime}(z=1)\right)^{2} .
$$

At $N>2$ the fixed point of this equation $R^{\prime}(z=1)=-\epsilon /[2(N-2)]<0$. It corresponds to the negative critical exponent $\eta(3.12)$ and hence is unphysical. However, we shall see that in the RA model some appropriate nonanalytical fixed points $R(z)$ appear. In these fixed points $R^{\prime \prime}(z=1)=\infty$. In Ref. 28] the RG charges are the derivatives of the function $R(z)$ at $z=1$. Thus, in a non-analytical fixed point these charges diverge. In the systems with a finite number of the charges their divergence implies the absence of a fixed point. However, if the number of the RG charges is infinite such a criterion does not work and is even ambiguous. Indeed, the set of charges can be chosen in different ways and e.g. the coefficients of the Taylor expansion about $z=0$ remain finite in our problem.

\section{RANDOM FIELD}

For the RF XY model the one-loop RG equations (3.8. 3.9) can be solved exactly [13]. The solution corresponds to QLRO with the critical exponents $\eta=\pi^{2} / 9 \epsilon, \gamma=1-\pi^{2} / 18 \epsilon$. In the first order in $\epsilon$ the exponent $\eta$ equals the prefactor $C$ before the logarithm in the correlation function 13] of the angles $\phi(\mathbf{x})$ between the spins $\mathbf{n}(\mathbf{x})$ and some fixed direction: $\left\langle\left(\phi\left(\mathbf{x}_{1}\right)-\phi\left(\mathbf{x}_{2}\right)\right)^{2}\right\rangle=C \ln \left|\mathbf{x}_{1}-\mathbf{x}_{2}\right|$. We expect that this coincidence does not extend to the higher orders.

If $N \neq 2$ the RG equation (3.9) is more complex. Fortunately, at $N>3$ there is still a simple method to study the large-distance behavior. The method is based on the Schwartz-Soffer inequality [25] and shows that QLRO is absent.

In Ref. 25] the inequality is proven for the Gaussian distribution of the random field. It can also be proved for the arbitrary RF distribution (Appendix A).

Let us demonstrate the absence of physically acceptable fixed points in the RF case at $N>3$. We derive some inequality for critical exponents. Then we show that the inequality has no solutions. We use a rigorous inequality for the connected and disconnected correlation functions 25]

$$
\langle\langle\mathbf{n}(\mathbf{q}) \mathbf{n}(-\mathbf{q})\rangle\rangle=\left\langle\mathbf{n}_{a}(\mathbf{q}) \mathbf{n}_{a}(-\mathbf{q})\right\rangle-\left\langle\mathbf{n}_{a}(\mathbf{q}) \mathbf{n}_{b}(-\mathbf{q})\right\rangle \leq \operatorname{const} \sqrt{\left\langle\mathbf{n}_{a}(\mathbf{q}) \mathbf{n}_{a}(-\mathbf{q})\right\rangle},
$$

where $\mathbf{n}(\mathbf{q})$ is a Fourier-component of the magnetization, $a, b$ are replica indices. The disconnected correlation function is described by the critical exponent (3.12). The large-distance behavior of the connected correlation function in a zero-temperature fixed point can be derived from the expression

$$
\chi \sim \int\langle\langle\mathbf{n}(\mathbf{0}) \mathbf{n}(\mathbf{x})\rangle\rangle d^{D} x
$$


and the critical exponent of the susceptibility (3.13). The integral in the right hand side of Eq. (4.2) is proportional to $R_{c}^{D-\eta_{1}}$, where $R_{c}$ is the correlation length in the external field $H, \eta_{1}$ the critical exponent of the connected correlation function. For the calculation of the exponent $\gamma(\sqrt{3.13})$ we used the zero-loop expression of $R_{c}$ via $H$. Now we need the one-loop accuracy. In this order $R_{c} \sim H^{-1 /\left[2-(N-3) R^{\prime \prime}(0)\right]}$. This allows us to get the following equation for the exponent $\eta_{1}$

$$
\eta_{1}=D-2-2 R^{\prime \prime}(0)
$$

In a fixed point Eq. (4.1) provides an inequality for the critical exponents of the connected and disconnected correlation functions [25]. The inequality has the form

$$
2\left(2-D+\eta_{1}\right) \geq 4-D+\eta
$$

This allow us to obtain the following relation

$$
4-D \leq \frac{3-N}{N-1} \eta+o(R)
$$

where $\eta$ is given by Eq. (3.12). The two-spin correlation function can not increase up to the infinity as the distance increases. Hence, the critical exponent $\eta$ is positive. At $N>3$ this is incompatible with Eq. (4.5) at small $\epsilon$. Thus, there are no accessible fixed points for $N>3$. This suggests the strong coupling regime with a presumably finite correlation length.

Certainly, in the RF XY model [12,13] Eq. (4.5) is satisfied. However, the unstable fixed points of the RG equations [13] do not satisfy the inequality.

The marginal Heisenberg case $N=3$ is the most difficult, since in the one-loop order the right hand side of Eq. (4.5) equals zero at $N=3$. Hence, the two-loop corrections may be relevant. The RF Heisenberg model is beyond the scope of the present paper.

\section{RANDOM ANISOTROPY}

In this section we investigate the possibility of QLRO in the RA $O(N)$ model. The first subsection is devoted to the simplest case of the XY model. The second subsection contains an inequality for the critical exponent $\eta$. The derivation of the inequality is analogous to Eq. (4.5). This inequality is applied in the next subsections. The third subsection contains the results for the Heisenberg model. In the last subsection we consider the case $N>3$.

$$
\text { A. } N=2
$$

This case is studied analogously to the RF XY model 13. At $N=2$ the RG equation (3.9) can be solved analytically. Its solution is a periodical function with period $\pi$. In interval $0<\phi<\pi$ the fixed point solution $R(\phi)$ is given by the formula

$$
R(\phi)=\frac{\pi^{4} \epsilon}{144}\left[1 / 36-(\phi / \pi)^{2}(1-(\phi / \pi))^{2}\right] .
$$

It is a stable fixed point. This can be verified with the linearization of the flow equation (3.9) for the small deviations from the fixed point. Another proof of the stability is based on the inequality of the next subsection.

The stable fixed point corresponds to the QLRO phase at low temperatures and weak disorder. The critical exponents $\eta=\pi^{2} \epsilon / 36, \gamma=1-\pi^{2} \epsilon / 72$.

The solution (5.1) is non-analytical at $\phi=0$, since $R^{I V}(\phi=0)=\infty$. Hence, the Taylor expansion over $\phi$ is absent. However, a power expansion over $|\phi|$ exists. We shall see below that the same behavior at small $\phi$ conserves also at other $N$.

\section{B. An inequality for a critical exponent}

We use the same approach as in the RF model. Since in the RA case the random field is conjugated with a second order polynomial of the magnetization, the Schwartz-Soffer inequality [25] should be applied to correlation functions 
of the field $m(\mathbf{x})=\left(n_{z}(\mathbf{x})\right)^{2}-1 / N$, where $n_{z}$ denotes one of the magnetization components, $1 / N$ is subtracted to ensure the relation $\langle m\rangle=0$.

To calculate the critical exponent $\mu$ of the disconnected correlation function we use the representation (3.2) and obtain the relation

$$
\left\langle m^{a}(\mathbf{x}) m^{a}\left(\mathbf{x}^{\prime}\right)\right\rangle=\left\langle m^{\prime a}(\mathbf{x}) m^{\prime a}\left(\mathbf{x}^{\prime}\right)\right\rangle\left(1-\frac{2 N \sum_{i}\left\langle\left(\phi_{i}^{a}\right)^{2}\right\rangle}{N-1}\right),
$$

where $a$ is a replica index, $m^{\prime}=\left(n_{z}^{\prime}\right)^{2}-1 / N$ the slow part of the field $m$. One finds $\mu=-4 N R^{\prime \prime}(0)$.

The critical exponent $\mu_{1}$ of the connected correlation function is determined analogously to the RF case. We apply a weak uniform field $\tilde{H}$, conjugated with the field $m$, and calculate the susceptibility $d m / d \tilde{H}$ in two ways. The result for the critical exponent is $\mu_{1}=D-2-2(N+2) R^{\prime \prime}(0)$.

The Schwartz-Soffer inequality provides a relation between the exponents $\mu$ and $\mu_{1}$. It has the same structure as Eq. (4.4). Finally, we obtain the following equation

$$
\eta \geq \frac{4-D}{4}(N-1)+o(R)
$$

In terms of the $\mathrm{RG}$ charge $R(\phi)$ this inequality can be rewritten in the form

$$
R^{\prime \prime}(0) \leq-\epsilon / 8+o(R)
$$

$$
\text { C. } N=3
$$

In this case we solve Eq. (3.9) numerically. Since coefficients of Eq. (3.9) are large as $\phi \rightarrow 0$, it is convenient to use a series expansion of the fixed-point solution $R(\phi)$ at small $\phi$. At the larger $\phi$ the equation can be integrated with the Runge-Kutta method. The following expansion over $t=\sqrt{(1-z) / 2}=|\sin (\phi / 2)|$ holds

$$
\begin{array}{r}
R(\phi) / \epsilon=\frac{(N-1) a^{2}}{1-4(N-2) a}+2 a \sin ^{2} \frac{\phi}{2} \pm \frac{4 \sqrt{2}}{3} \sqrt{\frac{-a+2(N-2) a^{2}}{N+2}}\left|\sin ^{3} \frac{\phi}{2}\right| \\
+\left(\frac{2 a}{3}-\frac{2}{3(N+4)}\right) \sin ^{4} \frac{\phi}{2}+O\left(\left|\sin ^{5} \frac{\phi}{2}\right|\right),
\end{array}
$$

where $a=R^{\prime \prime}(\phi=0) / \epsilon$. We see that the RG charge $R(\phi)$ is non-analytical at small $\phi$. Similar to the random manifold [15] and random field XY 13] models $R^{I V}(0)=\infty$.

Numerical calculations show that at any $N$ the solutions, compatible with the inequality (5.4), have sign "+" before the third term of Eq. (5.5). The solutions to be found are even periodical functions with period $\pi$. Hence, their derivative is zero at $\phi=\pi / 2$. At $N=3$ there is only one solution that satisfies Eq. (5.4). It corresponds to $R^{\prime \prime}(\phi=0)=-0.1543 \epsilon$. If this solution is stable Eqs. 3.12,3.13) provide the following results for the critical exponents

$$
\eta=0.62 \epsilon ; \gamma=1-0.15 \epsilon \text {. }
$$

All the other solutions of Eq. (3.9) do not satisfy Eq. (5.4) and hence are unstable.

We have still to test the stability of the solution found. For this aim we use an approximate method. First, we find an approximate analytical solution of Eq. (3.9). We rewrite Eq. (3.9), substituting $\omega\left(R^{\prime \prime}(\phi)\right)^{2}$ for $\left(R^{\prime \prime}(\phi)\right)^{2}$. The case of interest is $\omega=1$ but at $\omega=0$ the equation can be solved exactly. The solution at $\omega=1$ can then be found with the perturbation theory over $\omega$. The exact solution at $\omega=0$ is $R_{\omega=0}(\phi)=\epsilon(\cos 2 \phi / 24+1 / 120)$. The corrections of order $\omega^{k}$ are trigonometric polynomials of order $2(k+1)$. The first correction is

$$
R_{1}(\phi)=-\frac{2 \omega \epsilon}{99} \cos 2 \phi+\frac{\omega \epsilon}{264} \cos 4 \phi+\text { const }
$$

After the calculation of the corrections we can write an asymptotic series for the critical exponent $\eta(3.12): \eta=$ $\epsilon\left(0.67-0.08 \omega+0.14 \omega^{2}-\ldots\right)$. The resulting estimation $\eta=\epsilon(0.67 \pm 0.08)$ agrees with the numerical result $(5.6)$ well. This allows us to expect that the stability analysis of the solution $R_{\omega=0}$ of the equation with $\omega=0$ provides information about the stability of the solution of Eq. (3.9). 
To study the stability of the exact solution of the equation with $\omega=0$ is a simple problem. We introduce a small deviation $r(\phi): R(\phi)=R_{\omega=0}(\phi)+r(\phi)$ and write the flow equation for this deviation:

$$
\frac{d r(\phi)}{d \ln L}=\left(5 r(\phi)+r^{\prime \prime}(\phi)+r^{\prime \prime}(0) \cos 2 \phi\right) / 3+\text { const } \times r^{\prime \prime}(0) .
$$

It is convenient to use the Fourier expansion $r(\phi)=\sum_{m} a_{m} \cos 2 m \phi$. The flow equations for the Fourier harmonics can be easily integrated. We see that $a_{m} \rightarrow 0$ as $L \rightarrow \infty$ for any $m>0$. The solution is unstable with respect to the constant shift $a_{0}$. However, this instability has no interest for us, since the correlation functions do not change at such shifts 15. Indeed, the constant shift corresponds to the addition of just a random term, independent of the magnetization, to the Hamiltonian (2.1). Thus, the RG equation possesses a stable fixed point. This fixed point describes the QLRO phase with the critical exponents (5.6).

In the Abelian systems the results of the functional RG are supported by the variational method [16]. In our problem this method can not be applied. However, it is interesting that in the Abelian systems the functional RG equations without $\left(R^{\prime \prime}(\phi)\right)^{2}$ reproduce the variational results.

As usual in critical phenomena, in 4 dimensions the one-loop RG equations allow one to obtain the exact largedistance asymptotics of the correlation function. In the 4-dimensional case $R(\phi)=\tilde{R}(\phi) / \ln L$, where $\tilde{R}(\phi)$ satisfies Eq. (3.9) at $\epsilon=1$. We obtain the following result for the two-spin correlation function with Eq. (3.11)

$$
\left\langle\mathbf{n}(\mathbf{x}) \mathbf{n}\left(\mathbf{x}^{\prime}\right)\right\rangle \sim \ln ^{-0.62}\left|\mathbf{x}-\mathbf{x}^{\prime}\right| .
$$

$$
\text { D. } N>3
$$

Numerical analysis of Eq. (3.9) shows that solutions, compatible with Eq. (5.4), are absent at $N \geq 10$. Hence, QLRO is absent for any $N \geq 10$. In the spherical model $(N=\infty)$ the absence of fixed points can be demonstrated analytically (Appendix C). This agrees with the previous results [9,23]. For each integer $N<10$ the RG equation (3.9) has exactly one solution, satisfying the inequality of section VB. These solutions are described in Table I. In the table, $\eta$ is the critical exponent of the two-spin correlation function, $\Delta_{T}$ the scaling dimension of the temperature (3.10).

Unfortunately, it is not clear if the fixed points, found at $N>3$, survive in 3 dimensions. A zero-temperature fixed point can exist only if the scaling dimension of the temperature is negative. Table il shows that scaling dimension is positive in the one-loop approximation at $\epsilon=1$ and $N \geq 5$. In the 3-dimensional $O(4)$ model the one-loop correction to the scaling dimension $-2(N-2) R^{\prime \prime}(0) \approx 0.7 \epsilon$ is close to the zero-loop approximation $-2+\epsilon$. Thus, the next orders of the perturbation theory are crucial to understand what happens in 3 dimensions.

In the $O(2)$ model the scaling dimension $\Delta_{T}=-2+\epsilon$ is exact 13.15. Hence, QLRO disappears in 2 dimensions. In systems with a larger numbers of magnetization components fluctuations become stronger. Thus, one expects the absence of QLRO in all the two-dimensional $O(N)$ models.

At the zero temperature Eq. (3.9) is valid independently of the scaling dimension $\Delta_{T}$. It is tempting to assume that at the zero temperature QLRO still exists in the RA $O(N>3)$ models below the critical dimension, in which $\Delta_{T}=0$. However, the experience of the two-dimensional RF XY model does not support such an expectation. Recent numerical simulations show that QLRO is absent even in the ground state of that model [30].

TABLE I. Critical exponents of the RA $O(N)$ model.

\begin{tabular}{ccccccccc}
\hline \hline$N$ & 2 & 3 & 4 & 5 & 6 & 7 & 8 \\
\hline$\eta$ & $\pi^{2} \epsilon / 36$ & $0.62 \epsilon$ & $1.1 \epsilon$ & $1.7 \epsilon$ & $2.7 \epsilon$ & $4.6 \epsilon$ & $9.0 \epsilon$ & $33 \epsilon$ \\
$\Delta_{T}$ & $-2+\epsilon$ & $-2+1.3 \epsilon$ & $-2+1.7 \epsilon$ & $-2+2.3 \epsilon$ & $-2+3.2 \epsilon$ & $-2+4.8 \epsilon$ & $-2+8.7 \epsilon$ & $-2+30 \epsilon$ \\
\hline \hline
\end{tabular}




\section{CONCLUSION}

We have obtained QLRO in the RA Heisenberg model. This is the first example of QLRO in a non-Abelian system. The RF disorder tends to destroy the ordering which exists in the RA case. This difference between the RF and RA models is not surprising, since the same difference was already obtained in Ref. 17 for the two-dimensional RF and RA XY models with the dipole forces.

We have not yet discussed the role of the topological defects. The contribution of the topological excitations to the $\mathrm{RG}$ equations (3.8,3.9) is determined by the rare regions where the random field is sufficiently strong to compensate the core energy. Hence, similar to the pure system in $2+\epsilon$ dimensions they are responsible for the non-perturbative corrections of order $\exp (-1 / \epsilon)$. Thus, their effect is negligible at small $\epsilon$. Several studies were devoted to the role of the vortices in the RF XY model [31]. The theoretical prediction of QLRO in this system is based on the vortexless version of the model [12,13]. A qualitative estimation [13] and variational calculations [31] suggest that the topological defects do not change the behavior of the RF XY model at the weak disorder. Our approach allows us to consider the XY model, including vortices. We see that QLRO does exist in the model with the defects.

However, in our problem there may be a more important source of the non-perturbative corrections. The effect of the multiple energy minima can lead to corrections of order $\epsilon^{5 / 2}$ to the RG equations [15]. Unfortunately, the non-perturbative effects in the RF systems are not well understood.

The present paper uses a systematic RG approach. However, some results can be reproduced more simply with an approximate Migdal-Kadanoff renormalization group (Appendix B).

The question of the large-distance behavior of the RF and RA Heisenberg models was discussed in Ref. [20] on the basis of an approximate equation of state. In that paper QLRO was also obtained in the RA case. However, we believe that this is an accidental coincidence, since the equation of state [20] is valid only in the first order in the strength of the disorder, while higher orders are crucial for critical properties [24. In particular, the approach [20] incorrectly predicts the absence of QLRO in the RF XY model and its presence in the exactly solvable RA spherical model. It also provides incorrect critical exponents in the Heisenberg case. The reason of the mistakes is the fact that in the weak external uniform field the perturbation parameter of Ref. 20] is large.

The RA Heisenberg model is relevant for the amorphous magnets [2]. At the same time, for their large-distance behavior the dipole interaction may be important [21]. Besides, a weak nonrandom anisotropy is inevitably present due to mechanical stresses.

In conclusion, we have found that the random anisotropy Heisenberg model has an infinite correlation length and a power dependence of the correlation function of the magnetization on the distance at low temperatures and weak disorder in $4-\epsilon$ dimensions. On the other hand, the correlation length of the random field $O(N>3)$ model is always finite.

\section{ACKNOWLEDGMENTS}

The author thanks E. Domany, G. Falkovich, M.V. Feigelman, Y. Gefen, S.E. Korshunov, Y.B. Levinson, A.I. Larkin, V.L. Pokrovsky and A.V. Shytov for useful discussions. This work was supported by RFBR grant 96-0218985 and by grant 96-15-96756 of the Russian Program of Leading Scientific Schools.

\section{APPENDIX A: INEQUALITY FOR CORRELATION FUNCTIONS}

In this appendix we derive an inequality for the correlation functions of the disordered systems. We consider the system with the Hamiltonian

$$
H=\int d x^{D}\left[H_{1}(\phi(\mathbf{x}))-h(\mathbf{x}) m(\phi(\mathbf{x}))\right],
$$

where $\phi$ is the order parameter, $h$ the random field with short range correlations, $H_{1}$ may depend on some other random fields. We prove an inequality for the Fourier components of the field $m$ :

$$
G_{c o n}(\mathbf{q}) \leq \operatorname{const} \sqrt{G_{d i s}(\mathbf{q})}
$$

where $G_{\text {dis }}(\mathbf{q})=\overline{\langle\mathbf{m}(\mathbf{q}) \mathbf{m}(-\mathbf{q})\rangle}, G_{\text {con }}(\mathbf{q})=\overline{\langle\mathbf{m}(\mathbf{q}) \mathbf{m}(-\mathbf{q})\rangle}-\overline{\langle\mathbf{m}(\mathbf{q})\rangle\langle\mathbf{m}(-\mathbf{q})\rangle}$, the angular brackets denote the thermal averaging, the bar denotes the disorder averaging. 
This inequality can be easily obtained in the case of the Gaussian distribution $P(h)$ of the field $h$ [25]. Indeed, in the Gaussian case

$$
\begin{array}{r}
G_{d i s}(\mathbf{q})=\int\left(P(h) \frac{d}{d h(\mathbf{q})} m_{\mathbf{q}}(h)\right) D\{h\}= \\
-\int\left(\frac{d}{d h(\mathbf{q})} P(h) m_{\mathbf{q}}(h)\right) D\{h\}=\mathrm{const} \int\left(P(h) h(-\mathbf{q}) m_{\mathbf{q}}(h)\right) D\{h\},
\end{array}
$$

where $\int D\{h\}$ denotes the integration over the realizations of the random field, $m_{\mathbf{q}}(h)=\int D\{\phi\} \exp (-H / T) m(\mathbf{q}) /$ $\int D\{\phi\} \exp (-H / T)$. Applying the Cauchy-Bunyakovsky inequality to Eq. (A3) one gets Eq. (A2).

However, the assumption about the Gaussian distribution of the random field is not necessary. The inequality (A2) can also be extended to a more general situation, corresponding to the effective replica Hamiltonian (3.1). Indeed, if one adds to any Hamiltonian a weak Gaussian random field $\tilde{h}$, conjugated with the field $m$, it suffices for Eq. (A2) to become valid. The addition of the Gaussian random field corresponds to the transformation $R\left(\mathbf{n}_{a} \mathbf{n}_{b}\right) \rightarrow$ $R\left(\mathbf{n}_{a} \mathbf{n}_{b}\right)+\Delta \mathbf{n}_{a} \mathbf{n}_{b}$ in Eq. (3.1) where $\Delta \sim \tilde{h}^{2}$ is a positive constant. Thus, Eq. (A2) is invalid only, if for the arbitrarily small $\Delta$ the replica Hamiltonians can not contain the two-replica contribution $R\left(\mathbf{n}_{a} \mathbf{n}_{b}\right)=R\left(\mathbf{n}_{a} \mathbf{n}_{b}\right)-\Delta \mathbf{n}_{a} \mathbf{n}_{b}$. This corresponds to the border of the region of the possible Hamiltonians and has zero probability.

For systems in the critical domain there is a simple way to understand why the inequality is valid not only in the Gaussian case but also in the general situation. This is just a consequence of the universality.

\section{APPENDIX B: MIGDAL-KADANOFF RENORMALIZATION GROUP}

This appendix contains a simple approximate version of the renormalization group. The results for the critical exponents of the XY and Heisenberg models have a very good accuracy. The value of the magnetization component

number $N_{c}$, at which QLRO disappears in the RF model, is probably exact. However, the critical number of the components in the RA model is underestimated.

\section{Random field}

We use the following ansatz for the disorder-induced term in the Hamiltonian (2.1): $R\left(\mathbf{n}_{a} \mathbf{n}_{b}\right)=\alpha \mathbf{n}_{a} \mathbf{n}_{b}+\beta$, where $\alpha$ and $\beta$ are constants. This expression corresponds to the Gaussian RF disorder (2.2). Below we ignore the generation of the other contributions to the function $R(z)$. The missed contributions are related with random anisotropies of different orders. In terms of the angle variable $\phi(3.8,3.9)$

$$
R(\phi)=\alpha \cos \phi+\beta
$$

To ensure consistency we have to truncate the RG equation (3.9). We substitute the ansatz (B1) into Eq. (3.9) and retain only the terms, proportional to $\cos \phi$ or independent of $\phi$. This leads to the following $R G$ equation for the constant $\alpha(\mathrm{B} 1)$

$$
\frac{d \alpha}{d \ln L}=\epsilon \alpha+2 \alpha^{2}(N-3)
$$

For $N<3$ Eq. (B2) has a stable solution $\alpha=\epsilon /[2(3-N)]$. The critical exponent 3.12$)$ equals

$$
\eta=\frac{(N-1) \epsilon}{(3-N)} .
$$

At $N=2$ this result has less than ten percent difference with the systematic theory 13 . QLRO disappears at $N=3$. This is the same critical number which is found in section IV.

For $N>3$ a fixed point exists in $4+\epsilon$ dimensions. It describes the transition between the ferromagnetic and paramagnetic phases. In this fixed point the critical exponent (B3) satisfies the modified dimensional reduction hypothesis 32]. However, we believe that this is an artifact of the Migdal-Kadanoff approximation, since the correct value of the critical exponent differs form Eq. (B3). 


\section{Random anisotropy}

In this case we use the ansatz $R\left(\mathbf{n}_{a} \mathbf{n}_{b}\right)=A\left(\mathbf{n}_{a} \mathbf{n}_{b}\right)^{2}+B$. In terms of the variable $\phi(3.8,3.9) R(\phi)=\alpha \cos 2 \phi+\beta$. We again substitute our ansatz into Eq. (3.9) and retain the terms, proportional to $\cos 2 \phi$, and the terms, independent of $\phi$. The RG equation for the constant $\alpha$ has the form

$$
\frac{d \alpha}{d \ln L}=\epsilon \alpha+8(N-6) \alpha^{2}
$$

The fixed point solution of this equation is $\alpha=\epsilon /[8(6-N)]$. It describes the QLRO phase at $N<6$. At $N=3$ the function $R(\phi)=\alpha \cos 2 \phi+\beta$ is just $R_{\omega=0}$ of section $\mathrm{VC}$. The critical exponent of the two-spin correlation function is given by the following equation

$$
\eta=\frac{\epsilon(N-1)}{6-N}
$$

At $N=2,3$ this value is close to the results of the systematic approach (Table I).

\section{APPENDIX C: SPHERICAL MODEL}

In this appendix we consider the spherical RA model with the functional RG. We show that QLRO is absent in this model. In the spherical limit $N=\infty$ only the terms, proportional to $N$, and the term $\epsilon R(z)$ should be retained in the right hand side of Eq. (3.7). After the change of the variable $R(z)=\epsilon r(z) / N$ one obtains

$$
0=r(z)\left[1+4 r^{\prime}(1)\right]-2 z r^{\prime}(1) r^{\prime}(z)+\left(r^{\prime}(z)\right)^{2} .
$$

It is convenient to differentiate Eq. (C1) over $z$. One gets

$$
0=r^{\prime}(z)\left[1+2 r^{\prime}(1)\right]+2 r^{\prime \prime}(z)\left[r^{\prime}(z)-z r^{\prime}(1)\right] \text {. }
$$

Analytical functions $r(z)$ can satisfy Eq. (C2) at $z=1$ only if $r^{\prime}(1)=0$ or $r^{\prime}(1)=-1 / 2$. In both cases Eq. (C2) can be easily solved. There are three analytical non-zero solutions: $r(z)=-z / 2+1 / 4 ; r(z)=-(1-z)^{2} / 4 ; r(z)=-z^{2} / 4$. The last solution only has the necessary symmetry.

The non-analytical solutions are absent. Indeed, Eq. (C2) can be integrated with the substitution $r^{\prime}(z)=z t(z)$. The general integral has the form

$$
\frac{(t(z))^{2 t(1)}}{(2 t(z)+1)^{2 t(1)+1}}=C z .
$$

Besides, there are special solutions. They all satisfy the relation $t(z)=t(1)$. Hence, the special solutions are analytical. Thus, the function $t(z)$ can be non-analytical at $z=1$ only under the condition that $z=1$ is a peculiar point of Eq. (C3). This means that $t(1)=0$ or $t(1)=-1 / 2$. However, it is easy to verify that in both cases the solution is one of the found above.

We see that the only fixed point of the spherical RA model is $R(z)=-\epsilon z^{2} /(4 N)$. With Eq. (3.12) one finds the critical exponent $\eta=-\epsilon / 2$. Since $\eta>0$ the solution found is applicable at $D>4$. At $D<4$ the fixed points are absent. Thus, QLRO is absent too.

[1] Y. Imry and S.K. Ma, Phys. Rev. Lett. 35, 1399 (1975).

[2] R. Harris, M. Plischke, and M.J. Zuckermann, Phys. Rev. Lett. 31, 160 (1973).

[3] D.J. Sellmyer and M.J. O'Shea, in Recent Progress in Random Magnetism, ed. D. Ryan (World Scientific, Singapore, 1992) p. 71.

[4] N.A. Clark, T. Bellini, R.M. Malzbender, B.N. Thomas, A.G. Rappaport, C.D. Muzny, D.W. Shaefer, and L. Hrubesh, Phys. Rev. Lett. 71, 3505 (1993). T.Bellini, N.A. Clark, and D.W. Schaefer, Phys. Rev. Lett. 74, 2740 (1995). H. Haga and C.W. Garland, Liq. Cryst. 22, 275 (1997). 
[5] S.V. Fridrikh and E.M. Terentjev, Phys. Rev. Lett. 79, 4661 (1997). T. Emig, Phys. Rev. Lett. 82, C3380 (1999).

[6] J.V. Porto III and J.M. Parpia, Phys. Rev. Lett. 74, 4667 (1995). K. Matsumoto, J.V. Porto, L. Pollak, E.N. Smith, T.L. Ho, and J.M. Parpia, Phys. Rev. Lett. 79, 253 (1997).

[7] G. Blatter, M.V. Feigel'man, V.B. Geshkenbein, A.I. Larkin, V.M. Vinokur, Rev. Mod. Phys. 66, 1125 (1994).

[8] A.I. Larkin, Zh. Eksp. Teor. Fiz. 58, 1466 (1970) [Sov. Phys. JETP 31, 784 (1970)].

[9] R.A. Pelcovits, E. Pytte, and J. Rudnik, Phys. Rev. Lett. 40, 476 (1978).

[10] M. Aizeman and J. Wehr, Phys. Rev. Lett. 62, 2503 (1989); Commun. Math. Phys. 150, 489 (1990).

[11] U. Yaron, P.L. Gammel, D.A. Huse, R.N. Kleiman, C.S. Oglesby, E. Bucher, B. Batlogg, D.J. Bishop, K. Mortensen, K. Clausen, C.A. Bolle, and F. De La Cruz, Phys. Rev. Lett. 73, 2748 (1994).

[12] S.E. Korshunov, Phys. Rev. B 48, 3969 (1993).

[13] T. Giamarchi and P. Le Doussal, Phys. Rev. Lett. 72, 1530 (1994); Phys. Rev. B 52, 1242 (1995).

[14] M.J.P. Gingras and D.A. Huse, Phys. Rev. B 53, 15193 (1996).

[15] D.S. Fisher, Phys. Rev. Lett. 56, 1964 (1986). L. Balents and D.S. Fisher, Phys. Rev. B 48, 5949 (1993).

[16] M. Mezard and G. Parisi, J. Phys. A 23, L1229 (1990); J. Phys. I France 1, 809 (1991).

[17] D.E. Feldman, Pis'ma ZhETF 65, 108 (1997) [JETP Lett. 65, 114 (1997)]; Phys. Rev. B 56, 3167 (1997).

[18] T. Emig and T. Nattermann, Phys. Rev. Lett. 81, 1469 (1998); cond-mat/9810367. A. Hazareesing and J.-P. Bouchaud, cond-mat/9810097.

[19] L. Radzihovsky and J. Toner, cond-mat/9811105.

[20] A. Aharony and E. Pytte, Phys. Rev. Lett. 45, 1583 (1980).

[21] B. Barbara, M. Coauch, and B. Dieny, Europhys. Lett. 3, 1129 (1987).

[22] R. Fisch, Phys. Rev. B 57, 269 (1998); ibid 58, 5684 (1998). J. Chakrabaty, Phys. Rev. Lett. 81, 385 (1998).

[23] P. Lacour-Gayet and G. Toulouse, J. Phys. (Paris) 35, 425 (1974). S.L. Ginzburg, Zh. Eksp. Teor. Fiz. 80, 244 (1981). A. Khurana, A. Jagannathan, and J.M. Kosterlitz, Nucl. Phys. B 240, 1 (1984). M.V. Feigelman and M.V. Tsodyks. Zh. Eksp. Teor. Fiz. 91, 955 (1986) [Sov. Phys. JETP 64, 562 (1986)].

[24] Y.Y. Goldshmidt, Nucl. Phys. B 225, 123 (1983).

[25] M. Schwartz and A. Soffer, Phys. Rev. Lett. 55, 2499 (1985).

[26] A.M. Polyakov, Phys. Lett. 59B, 79 (1975); Gauge Fields and Strings (Harwood Academic Publishers, Chur, 1987).

[27] The results about QLRO, obtained for the RF XY model in Ref. 12,13, can be easily extended to the RA XY model, since in terms of the angles $\phi(\mathbf{x})$ between the spins $\mathbf{n}(\mathbf{x})$ and some fixed direction the Hamiltonians of these models are almost identical.

[28] D.S. Fisher, Phys. Rev. B 31, 7233 (1985).

[29] J. Zinn-Justin, Quantum Field Theory and Critical Phenomena (Oxford University Press, Oxford, 1993).

[30] C. Zeng, P.L. Leath, and D.S. Fisher, cond-mat/9807281.

[31] T. Garel, G. Iori, and H. Orland, Phys. Rev. B 53, R2941 (1996). D. Carpentier, P. Le Doussal, and T. Giamarchi, Europhys. Lett. 35, 379 (1996). J. Kierfeld, T. Nattermann, and T. Hwa, Phys. Rev. B 55, 626 (1997).

[32] M. Schwartz and A. Soffer, Phys. Rev. B 33, 2059 (1986). 